\title{
Adaptive Spatio-temporal Filtering of 4D CT-Heart
}

\author{
Mats Andersson and Hans Knutsson \\ Division of Medical Informatics, Department of Biomedical Engineering and \\ Center for Medical Image Science and Visualization (CMIV) \\ Linköping University, Linköping, Sweden \\ \{matsa, knutte\}@imt.liu.se \\ www.imt.liu.se/mi
}

\begin{abstract}
The aim of this project is to keep the x-ray exposure of the patient as low as reasonably achievable while improving the diagnostic image quality for the radiologist. The means to achieve these goals is to develop and evaluate an efficient adaptive filtering (denoising/image enhancement) method that fully explores true 4D image acquisition modes.

The proposed prototype system uses a novel filter set having directional filter responses being monomials. The monomial filter concept is used both for estimation of local structure and for the anisotropic adaptive filtering. Initial tests on clinical 4D CT-heart data with ECG-gated exposure has resulted in a significant reduction of the noise level and an increased detail compared to $2 \mathrm{D}$ and $3 \mathrm{D}$ methods. Another promising feature is that the reconstruction induced streak artifacts which generally occur in low dose CT are remarkably reduced in $4 \mathrm{D}$.
\end{abstract}

Keywords: adaptive filtering, 4D image denoising, low dose CT, monomial filters.

\section{Introduction}

A problem of central importance in medical imaging is the balance between image quality and ionizing radiation dose. A number of approaches can be attempted to address this problem: Refined exposure control: A technique available in many clinical scanners today is modulation of the tube current during the rotation, so that the dose decreases in those slices and at those angular positions where lower attenuation is expected and full exposure is not needed. In ECG-gated cardiac CT, the tube current can be modulated during the cardiac cycle with reduced radiation exposure during the systolic phase. Filtering the reconstructed images: Applying filters after image reconstruction has not been widely used in $3 \mathrm{D}$ (or 4D). In spite of the fact that most cardiac CT-scanners generate 3D or $4 \mathrm{D}$ data the examination of the multidimensional data is generally performed by examination of a series of $2 \mathrm{D}$ images. Most events in $4 \mathrm{D}$ data have a structure that is one or two dimensional. The local structure of a moving heart wall is intrinsically one dimensional (the 4D local structure tensor has rank 1) and a tube structure like a vessel is two dimensional. Using true $4 \mathrm{D}$ denoising the remaining dimensions provide a powerful base to filter out the noise. 
In relation to more recent methods such as e.g. non local means [5] and bilateral filtering [7] adaptive filtering is here used for a 4D prototype system for medical image enhancement for three reasons. 1) Computational efficiency. 2) The tuning process is more or less straight forward. 3) The most important reason is however that adaptive filtering has proved to bee extremely robust and is widely used in clinical 2D (and 3D) applications.

\section{The Power of Dimensionality, an Initial Experiment}

In this experiment a synthetic 4D image is used to illustrate the power of adaptive filtering with respect to dimensionality. To the top left in figure 1 a $2 \mathrm{D}$ image out of the synthetic $4 \mathrm{D}$ volume is shown. The size of the $4 \mathrm{D}$ volume is $127 \times 127 \times 9 \times 9$ and the $4 \mathrm{D}$ test image has no signal variation in the last two dimensions. There are three events that can be observed in this image: a large step close to the diagonal, a thin white line and a shading from the upper left corner to the lower right corner. Next the test image is degraded with a massive amount of $4 \mathrm{D}$ additive noise. As all events in the test image share the same orientation the adaptive filtering parameters can be set globally. The top right part of figure 1 shows the $4 \mathrm{D}$ filter kernel. The filter plot consists of $9 \times 9$ tiles where each tile is of size $9 \times 9$ pixels. The first two $4 \mathrm{D}$ coordinates of the filter kernel specify one of these tiles, the remaining two $4 \mathrm{D}$ coordinates refers to the position within this tile.

By using corresponding subsets of the $4 \mathrm{D}$ data and the $4 \mathrm{D}$ filter, the result from a 2D, a 3D and a 4D adaptive filtering approach is computed, figure 1. For the $2 \mathrm{D}$ case a massive amount of noise remains, but some faint traces of the edge
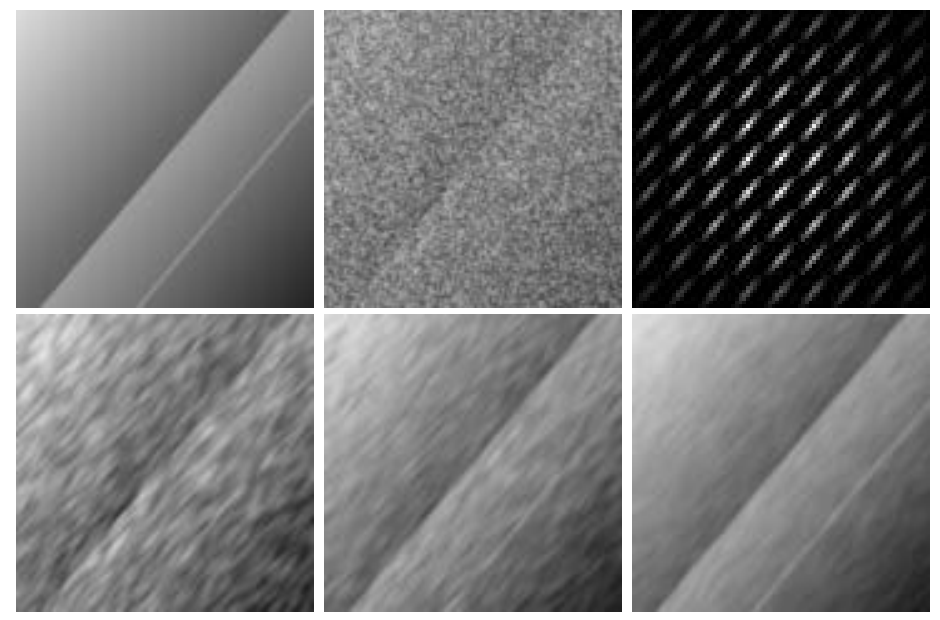

Fig. 1. Top: a 2D slice of the synthetic 4D image, test image after degradation by $4 \mathrm{D}$ noise, the global 4D enhancement filter kernel of size $9^{4}$. Bottom: Result after 2D, 3D and $4 \mathrm{D}$ adaptive filtering. 
and the shading can be discerned. In 3D the noise level is significantly reduced but the white line is not discernible. In the $4 \mathrm{D}$ case the thin white line is clearly visible and the nose is significantly reduced compared to $3 \mathrm{D}$.

\section{4D Adaptive Filtering}

The basic principle behind adaptive filtering is to use a local anisotropic filter synthesis originally referred to as steerable or controllable filters, 2 2120]. The method stresses the importance of locally 1D structures and was first implemented for 2D images [17 and was later applied to 3D signals (volumes and image sequences) in [15 10. The 4D adaptive filtering method proposed here is in some aspects a direct generalization of the concept for $2 \mathrm{D}$ and $3 \mathrm{D}$ adaptive filtering described in [10]. There are, however, certain features that has to be considered in the 4D case such as e.g. estimation of local structure and control of anisotropic features in the adaptive filtering.

The local structure is generally represented by using a second order tensor. Local structure analysis algorithms are quite complex and involve a lot more than the filters used. This makes comparisons difficult to interpret 1119]. Many frequently used structure tensor estimation algorithms can be defined as subsets of the monomial quadrature tensor proposed here, for example, the gradient tensor [34, the boundary tensor [18, the energy tensor 9], spatial 2:nd order polynome tensor 8 .

The authors stress the importance of the magnitude of the local structure tensor to be invariant to the phase of the signal. Alternative methods to compute a $4 \mathrm{D}$ structure tensor can be used within the proposed concept and the control tensor mappings below is valid for all structure tensors. Note however that the definition of the monomial filters are of interest not only for estimation of local structure but also for the directional properties of the adaptive filter set as the computation of the resulting image becomes extremely simple this way, see eq. 11.

The original phase invariant structure tensor $\mathbf{T}$, first proposed in [12 13], is computed from the magnitude of quadrature filter responses $q_{k}$ as $\mathbf{T}=q_{k} \mathbf{M}_{k}$ where the projection tensors $\mathbf{M}_{k}$ are computed from the orientation of the quadrature filters. In $4 \mathrm{D}$ this approach requires 10 or more quadrature filters evenly distributed according to a regular polyhedron over one half of the Fourier space. The only possible alternative is here the 24-cell which corresponds to twelve 4D filter kernels. Using 10 or more complex valued 4D filters requires an extreme computational effort for an evaluation on clinical data.

An alternative method to compute a local structure tensor while maintaining the phase invariant property is to use monomial filters. The directional part here consists of monomials i.e. products of positive integer powers of the components of $\hat{\boldsymbol{u}}$. Performing $n$ repeated outer products of $\hat{\boldsymbol{u}}$ will contain all order $n$ component products.

$$
\hat{\boldsymbol{u}}^{\otimes n}=\underbrace{\hat{\boldsymbol{u}} \otimes \hat{\boldsymbol{u}} \ldots \otimes \hat{\boldsymbol{u}}}_{n \text { entities }}
$$


Consider first a simple 2D example where $\hat{\boldsymbol{u}}=(u, v)^{T}$ the monomial filter response matrix of order one and two is for a simple signal with phase $\theta$ expressed as:

$$
\mathbf{Q}_{1}=-i \sin (\theta) \mathrm{A}_{\circ}(u, v)^{T} \quad \mathbf{Q}_{2}=\cos (\theta) \mathrm{A}_{\circ}\left(\begin{array}{cc}
u^{2} & u v \\
u v & v^{2}
\end{array}\right)
$$

Computing the outer products of the filter response matrices and using the fact that $u^{2}+v^{2}=1$ results in

$$
\mathbf{Q}_{1} \mathbf{Q}_{1}^{T}=\sin ^{2}(\theta)\left|\mathrm{A}_{\circ}\right|^{2}\left(\begin{array}{cc}
u^{2} & u v \\
u v & v^{2}
\end{array}\right) \quad \mathbf{Q}_{2} \mathbf{Q}_{2}^{T}=\cos ^{2}(\theta)\left|\mathbf{A}_{\circ}\right|^{2}\left(\begin{array}{cc}
u^{2} & u v \\
u v & v^{2}
\end{array}\right)
$$

Finally the phase invariant second order $2 \mathrm{D}$ tensor $\mathbf{T}$ is obtained as:

$$
\mathbf{T}=\mathbf{Q}_{1} \mathbf{Q}_{1}^{T}+\mathbf{Q}_{2} \mathbf{Q}_{2}^{T}=\left|\mathrm{A}_{\circ}\right|^{2}\left(\begin{array}{cc}
u^{2} & u v \\
u v & v^{2}
\end{array}\right)
$$

where $\mathrm{A}_{\circ}$ is a function of the radial filter function $R(\rho)$ and the signal amplitude. The monomial filter matrices of order one and two are in the FD defined as

$$
\boldsymbol{F}_{1 n}=R(\rho) \hat{\boldsymbol{u}}_{n} \quad \boldsymbol{F}_{2 m n}=R(\rho) \hat{\boldsymbol{u}}_{m} \hat{\boldsymbol{u}}_{n}
$$

Note that although $\boldsymbol{F}_{2}$ contain four entries the required number of second order filters are three in 2D. Using filters of order one and two is the most computationally efficient way compute a phase independent structure tensor from monomial filters. The $2 \mathrm{D}$ concept above can be directly generalized to higher dimensions and it is also possible to use higher order monomial filters, see [16]

In $4 \mathrm{D}$ the monomial filters of order one and two corresponds to 4 and 10 ( real valued) filters respectively compared to 12 complex valued filter in the quadrature approach. In addition, due to more smooth directional properties, monomial filters require a smaller spatial support compared to quadrature filters using the same radial filter function in the FD which helps to preserve detail and contrast in the processing. The radial filter function of the monomial filters $R(\rho)$ is lognormal with center frequency $u_{0}=3 \pi / 5$ (where $\pi$ is the Nyquist frequency)
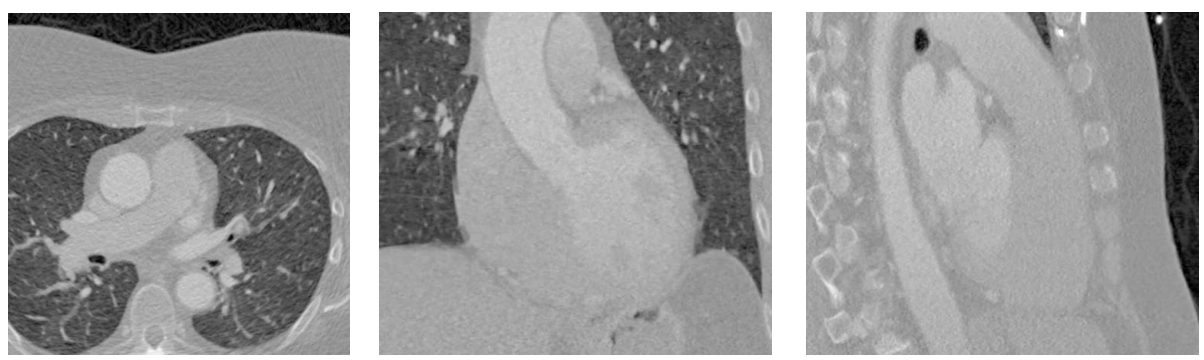

Fig. 2. Original 4D data set, from left to right: axial, frontal and sagittal 2D planes from low exposure part of heart cycle 
and a relative bandwidth $\beta=2.5$ octaves. The filter kernels are optimized with respect to, ideal frequency response, spatial locality and expected SNR 14. The clinical 4D data set is sampled in 20 points over the heart cycle. In relation to the spatial resolution of $0.75 \mathrm{~mm}^{3}$ voxels the temporal component $\left(u_{4}\right)$ is considered to be under sampled by a factor of 3 . To partly compensate for this under sampling the $u_{4}$ component of the filters is scaled by a factor of $1 / 3$. This implies that the radius in the FD is computed as $\rho=\sqrt{u_{1}^{2}+u_{2}^{2}+u_{3}^{2}+1 / 9 u_{4}^{2}}$. The 4D coronar angio data set was captured using a Siemens SOMATOM Definition. The size of the $4 \mathrm{D}$ data set is $512 \times 512 \times 446 \times 204 \mathrm{D}$ voxels. During the image acquisition the tube current is modulated over the cardiac cycle with reduced radiation exposure during the systolic phase. In figure 2 an axial, frontal and sagittal $2 \mathrm{D}$ slice of the $4 \mathrm{D}$ data set is shown for a low exposure part of the heart cycle.

\subsection{The Control Tensor, Mapping of Local Energy}

A local structure second order tensor in $4 \mathrm{D}$, computed according to eq. 4 , is positive definite and can be expressed as:

$$
\mathbf{T}=\sum_{k=1}^{4} \lambda_{k} \hat{\mathbf{e}}_{k} \hat{\mathbf{e}}_{k}^{T} \quad \lambda_{1} \geq \lambda_{2} \geq \lambda_{3} \geq \lambda_{4} \geq 0
$$

The tensor mimics the energy distribution in the FD and can pictured as a $4 \mathrm{D}$ ellipsoid where the extensions along the main axes, $\hat{\mathbf{e}}_{k}$, are defined by the corresponding eigen values $\lambda_{k}$. The eigen system defines the orientation of the ellipsoid and the tensor magnitude $\|\mathbf{T}\|$ determines the amount of energy (or contrast) in the local neighborhood. The result of the adaptive filtering is based on a set of second order monomial filter responses. We denote the filter response matrix of these enhancement filters $\mathbf{H}_{2}$. These filters are of high pass type and the radial filter function tapers down to zero above the Nyquist frequency, (note that the distance from the origin to a corner in the FD is $2 \pi$ in $4 \mathrm{D}$ ). For each neighborhood a result is computed as a weighted summation of the high pass filters plus one isotropic lowpass filter response, $\mathrm{h}_{0}$.

For smooth neighborhoods only the low pass filter is used. In this case the low pass filter provide an efficient increase in the SNR without degrading any structural information. When structure is present, it is on the other hand, of outmost importance to maintain the high pass information in one ore more orientations depending of the anisotrophy of the neighborhood. The core of the adaptive filtering is to define how much of each of the high pass filter responses to use for each individual neighborhood. For this purpose a control tensor, C, is introduced. The control tensor and the local structure tensor share the same eigen system but may differ in respect to magnitude and eigen value relations. The mapping $\mathbf{T}$ to $\mathbf{C}$ is consequently made to minimize the distance between the resulting adaptive filter and a local Wiener filter.

The mapping of local energy is performed according the $\gamma$-function to the top left plot in figure 3. The $\mathrm{x}$-axis of this plot corresponds to the tensor magnitude, 

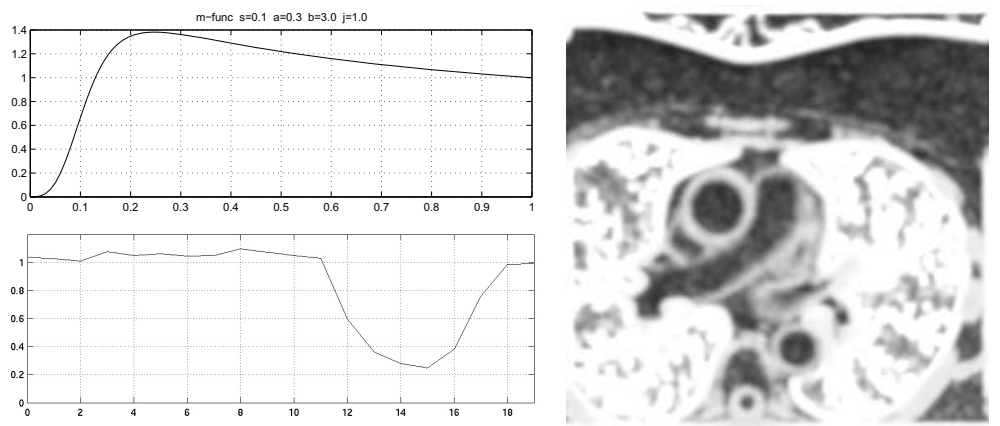

Fig. 3. Top left: $\gamma$-map of local energy. Lower left: Estimated noise variance over the heart cycle. Right: Resulting high pass map, the $\gamma$-image. See test for details.

$\sqrt{\operatorname{tr}(\mathbf{T}) /|\operatorname{tr}(\mathbf{T})|_{\max }}$, where the square root is due to the fact that the tensor is quadratic with respect to the image intensity, see eq. 4. The low end of the curve corresponds to a soft noise-threshold which is related to the estimated SNR ratio of the image. In areas where the energy is below the threshold no high pass filter will contribute substantially to the resulting image. The local energy contribution in the mid-range of the $\gamma$-function corresponds to faint structures in the image just above the noise threshold. The overshoot causes a compression which increase the visibility of these weak structures. The right end of the $\gamma-$ function corresponds to high contrast events that are clearly visible without further amplification.

The tuning processes of adaptive filtering is a matter of exploring optimal mapping parameters to the present image. For the magnitude mapping the most crucial parameter is the noise threshold. For the present CT-data the mapping of the noise threshold is further complicated by two facts. 1) The tube current is modulated over the heart cycle. The lower right part of figure 3 shows the estimated relative noise variance over the heart cycle. 2) Due to the nature of X-ray imaging the SNR also varies with the local intensity. Both these features are here compensated by a local adaptation of the noise threshold, i.e. the low end of the $\gamma$-function in figure 3. The right part of figure 3 corresponds to the mapping of the local energy for the axial slice of the test image. The intensity of the $\gamma$-image defines the amount of high pass energy that will be contributed to the result.

\subsection{The Control Tensor, Mapping of Local Anisotropy}

The shape of the local tensor is determined by the relation of the eigen values. In many applications the pairwise relation of the eigen values, $\lambda_{k+1} / \lambda_{k}$, is used in the accessing and mapping of anisotropy. As opposed to $2 \mathrm{D}$ and $3 \mathrm{D}$ such eigenvalue relations are more cumbersome in $4 \mathrm{D}$ as there is no direct way to solve the secular equation. For this reason an alternative way to efficiently map the degree of anisotropy in the denoising, based directly on the tensor components, is proposed. 

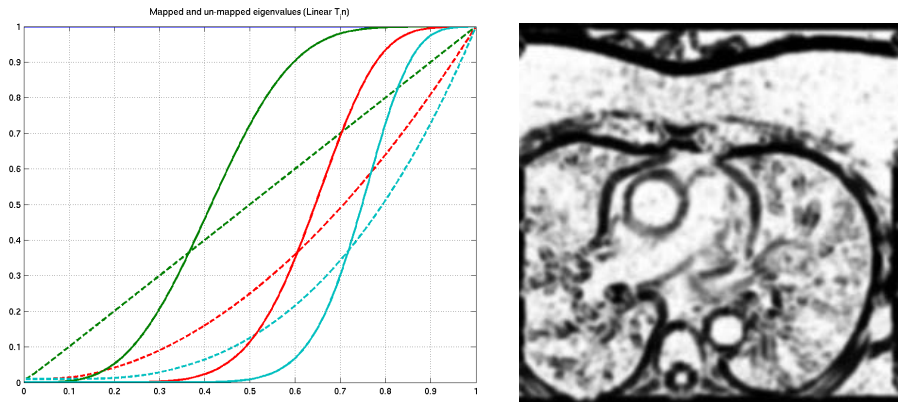

Fig. 4. Left: Resulting mapping of anisotropy $\left(\lambda_{k+1} / \lambda_{k}\right)$ for $p=4, n=2$ and $m=4$. Right: resulting energy independent anisotropy mapping, $\operatorname{tr}(\mathbf{C})$.

The anisotropic properties are mediated trough the control tensor, C. The control tensor is computed from the local structure tensor, $\mathbf{T}$, in an number of steps. The initial step is to compute

$$
\mathbf{C}_{1}=\mathbf{T}\left\|\mathbf{T}^{p}\right\|^{-\frac{1}{p}}
$$

The purpose here is to approximate a normalization of the control tensor with respect to $\lambda_{1}$ in eq. 6. Computing eq. 7 for $p=2^{n}$ is an efficient operation and for this case $p=4$ or $p=8$ is sufficient to put $\lambda_{1}$ in the desired range. Next we use the fact that $\mathbf{C}_{1}$ and $\mathbf{I}-\mathbf{C}_{1}$, where $\mathbf{I}$ is the identity matrix, both are positive definite and share the same eigen system.

$$
\mathbf{C}_{2}=\Theta\left(n, \mathbf{C}_{1}\right)=\mathbf{C}_{1}^{n}+n \mathbf{C}_{1}^{n}\left(\mathbf{I}-\mathbf{C}_{1}\right)
$$

The $\Theta$-mapping introduce a desired monotone sigma shaped mapping of the $\lambda_{k+1} / \lambda_{k}$ ratio in $\mathbf{C}_{2}$. The impact of this mapping is however considerable larger for the low end of $\lambda_{k+1} / \lambda_{k}$ ratio. To obtain a corresponding control of the upper end of the $\lambda_{k+1} / \lambda_{k}$ mapping we once more use the fact that $\mathbf{C}_{2}$ and $\mathbf{I}-\mathbf{C}_{2}$ share the same eigen system and compute:

$$
\mathbf{C}_{3}=\mathbf{I}-\Theta\left(m, \mathbf{I}-\mathbf{C}_{2}\right)
$$

Note that both the mappings of eq. 8 and eq. 9 use exponents that are powers of two (typically 4 and 8) which are efficient to compute. Figure 4 shows the effect of the proposed anisotropy mapping. The three dashed curves show $\lambda_{k+1} / \lambda_{k}$ for $\mathbf{C}_{1}$ and the solid curves show the resulting relation for $\mathbf{C}_{3}$ using $p=4, n=2$ and $m=4$. Note that so far this mapping is limited to the shape of the control tensor. The final control tensor $\mathbf{C}$ is calculated by merging the shape mapping of eq. 7. 9 by the $\gamma$-mapping of the local tensor magnitude from the previous section.

$$
\mathbf{C}=\gamma \mathbf{C}_{3}
$$


The denoised 4D image $\mathrm{J}$ can now be computed by the inner product of the control tensor $\mathbf{C}$, and the filter response matrix, $\mathbf{H}_{2}$, of the second order monomial high pass filters and adding the filter response of the low pass filter $h_{0}$.

$$
\mathrm{J}=\mathrm{h}_{0}+\left\langle\mathbf{C}, \mathbf{H}_{2}\right\rangle
$$

\subsection{Result}

Upper part of figure 5 shows the result of the 4D adaptive filtering from a normal dose part of the heart cycle. Note that the tuning here is extremely cautious but the result has a clearly improved detail and contrast. The main focus in the tuning process was here to level out the disturbing variation in SNR induced by the gated exposure. The lower part of figure 5 shows the result from a low dose part of the heart cycle. Note the difference in SNR between the
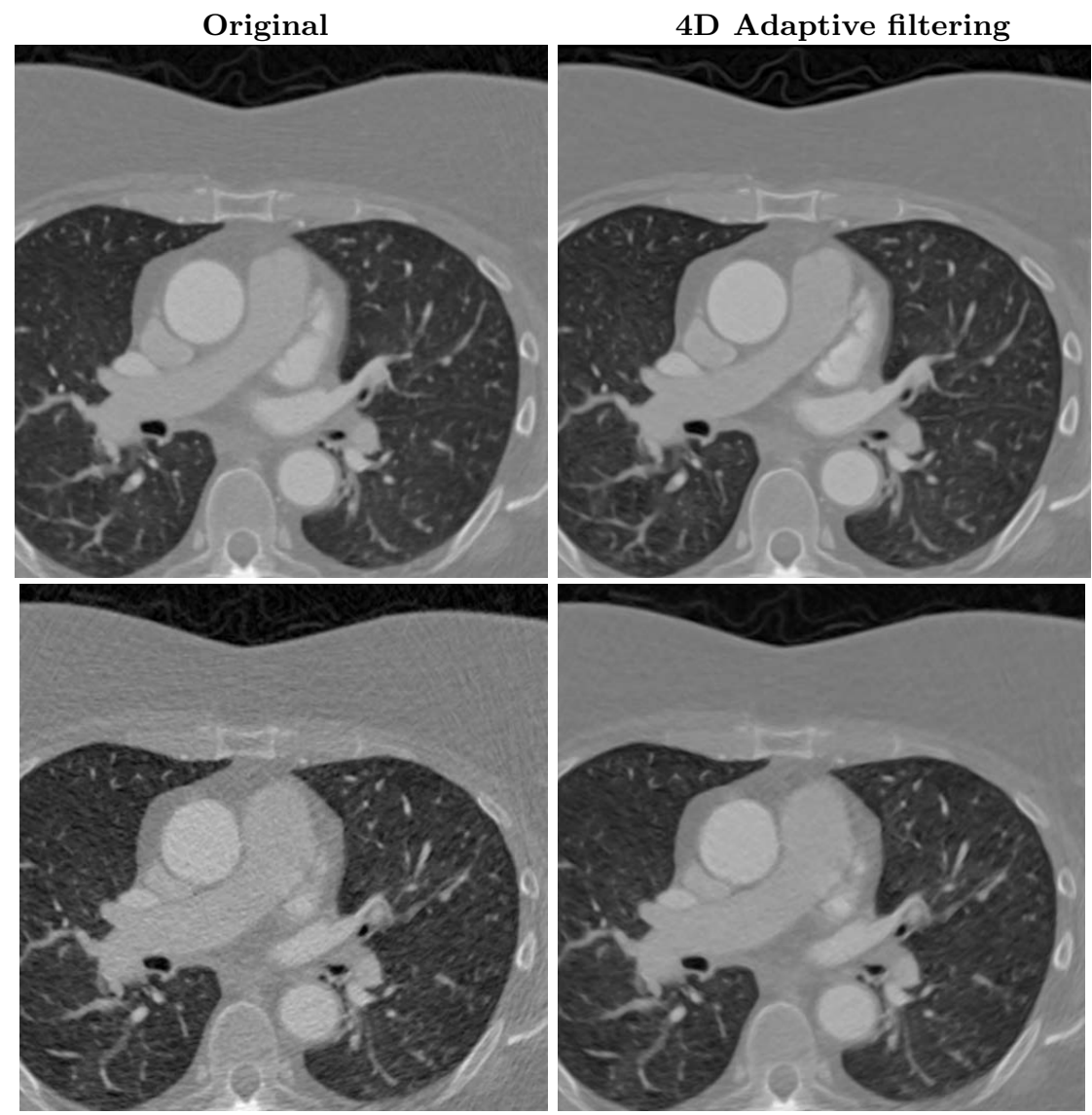

Fig. 5. Result from of $4 \mathrm{D}$ adaptive filtering. Upper: from a normal dose part of the heart cycle. Lower: result from a low dose part of the heart cycle. 

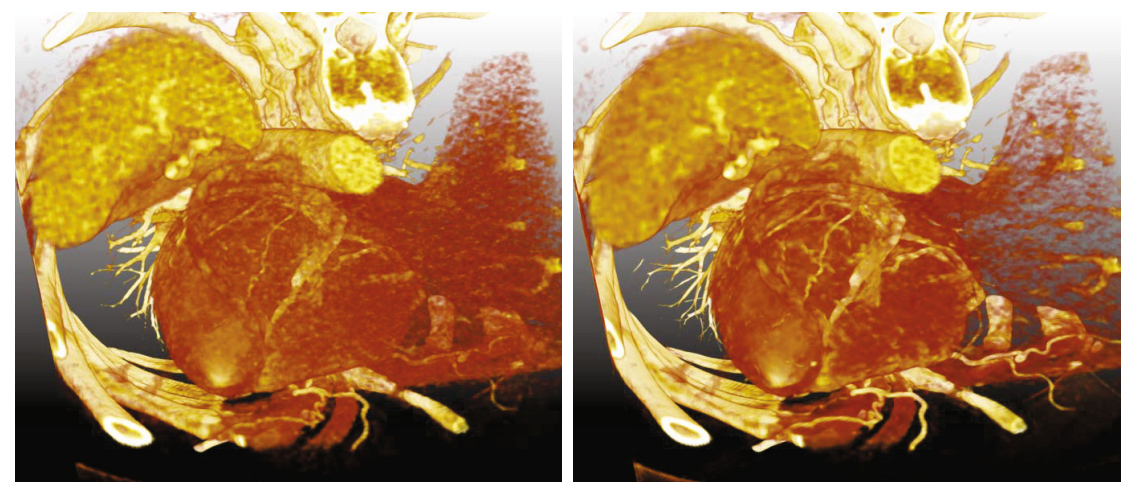

Fig. 6. Volume rendering of original (left) and $4 \mathrm{D}$ denoised (right) CT-heart from a normal exposure part of heart cycle. Note the increased visibility and detail of the coronary arteries and other vessels.

original images (left) and the result of the adaptive filtering (right). Also note that the reconstruction induced streaks which are mandatory in low dose CT are significantly reduced. This effect is most likely due to the fact that the streaks vary slightly over time due to motions of the heart and the patient.

In figure 6 a rendering of the original data and the result of the $4 \mathrm{D}$ algorithm is illustrated. These images are from the low exposure part of the heart cycle. The setting of the volume renderer is identical for both images. Note the improved visibility of the coronary arteries and the surrounding vessels.

Acknowledgments. This project is supported by the Swedish Research Council, grant 2008-3813 and 2011-5176 which are gratefully acknowledged. The authors would like to thank Örjan Smedby, Michael Sandborg and Chunliang Wang at the Department of Medical and Health Sciences, IMH/CMIV at Linköping University Hospital for providing anonymous clinical image data and for helpful comments in the tuning of the algorithm.

\section{References}

1. Andersson, M.: Controllable Multidimensional Filters in Low Level Computer Vision. Ph.D. thesis, Linköping University, Sweden, SE-581 83 Linköping, Sweden (September 1992), dissertation No 282, ISBN 91-7870-981-4

2. Bigün, J.: Recognition of local symmetries in gray value images by harmonic functions. In: The 9th International Conference on Pattern Recognition, Rome, Italy (1988)

3. Bigün, J., Granlund, G.H.: Optimal orientation detection of linear symmetry. In: IEEE First International Conference on Computer Vision, London, Great Britain, pp. 433-438 (1987)

4. Bigün, J., Granlund, G.H.: Optical flow based on the inertia matrix of the frequency domain. In: Proceedings from SSAB Symposium on Picture Processing, SSAB, Lund University, Sweden (1988) 
5. Buades, A., Coll, B., Morel, J.M.: A review of image denoising algorithms, with a new one. Multiscale Model Simul. 4, 490-530 (2005)

6. Eklund, A., Andersson, M., Knutsson, H.: True 4D image denoising on the GPU. International Journal of Biomedical Imaging, Article ID 952819 (2011)

7. Elad, M.: On the origin of the bilateral filter and ways to improve it. IEEE Transactions on Image Processing II(10), 1141-1151 (2003)

8. Farnebäck, G.: Polynomial Expansion for Orientation and Motion Estimation. Ph.D. thesis, Linköping University, Sweden, SE-581 83 Linköping, Sweden (2002) dissertation No 790, ISBN 91-7373-475-6

9. Felsberg, M., Jonsson, E.: Energy tensors: Quadratic, phase invariant image operators. In: Kropatsch, W.G., Sablatnig, R., Hanbury, A. (eds.) DAGM 2005. LNCS, vol. 3663, pp. 493-500. Springer, Heidelberg (2005)

10. Granlund, G.H., Knutsson, H.: Signal Processing for Computer Vision. Kluwer Academic Publishers (1995) ISBN 0-7923-9530-1

11. Johansson, B., Farnebäck, G.: A theoretical comparison of different orientation tensors. In: Proceedings SSAB 2002 Symposium on Image Analysis, SSAB, Lund, pp. 69-73 (2002)

12. Knutsson, H.: A tensor representation of 3-D structures. In: 5th IEEE-ASSP and EURASIP Workshop on Multidimensional Signal Processing, Noordwijkerhout, The Netherlands (September 1987) (poster presentation)

13. Knutsson, H.: Representing local structure using tensors. In: The 6th Scandinavian Conference on Image Analysis, Oulu, Finland, pp. 244-251 (June 1989); report LiTH-ISY-I-1019, Computer Vision Laboratory, Linköping University, Sweden (1989)

14. Knutsson, H., Andersson, M., Wiklund, J.: Advanced filter design. In: SCIA11. SCIA, Greenland (June 1999)

15. Knutsson, H., Haglund, L., Granlund, G.: Adaptive filtering of image sequences and volumes. In: Proceedings of International Conference on Automation, Robotics and Computer Vision, Singapore (September 1992)

16. Knutsson, H., Westin, C.F., Andersson, M.: Structure tensor estimation - introducing monomial quadrature filter sets. In: Laidlaw, D., Vilanova, A. (eds.) New Developments in the Visualization and Processing of Tensor Fields. Springer, Dagstuhl (2012)

17. Knutsson, H., Wilson, R., Granlund, G.H.: Anisotropic non-stationary image estimation and its applications - Part I: Restoration of noisy images. IEEE Transactions on Communications 31(3), 388-397 (1983)

18. Köthe, U.: Inegrated edge and junction detection with the boundary tensor. In: Proceedings of Ninth IEEE International Conference on Computer Vision (ICCV) (2003)

19. Nordberg, K., Farnebäck, G.: Estimation of orientation tensors for simple signals by means of second-order filters. Signal Processing: Image Communication 20(6), 582-594 (2005)

20. Perona, P.: Steerable-scalable kernels for edge detection and junction analysis. In: Sandini, G. (ed.) ECCV 1992. LNCS, vol. 588, pp. 3-18. Springer, Heidelberg (1992) 